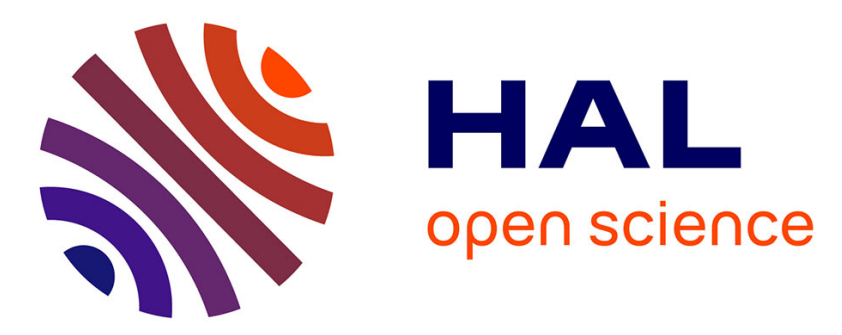

\title{
Linear viscoelasticity of an acrylate IPN, analysis and micromechanical modeling
}

\author{
Julie Diani, Éléonore Strauch-Hausser
}

\section{To cite this version:}

Julie Diani, Éléonore Strauch-Hausser. Linear viscoelasticity of an acrylate IPN, analysis and micromechanical modeling. Soft Matter, 2021, 17 (31), pp.7341 - 7349. 10.1039/d1sm00808k . hal-03320517

\section{HAL Id: hal-03320517 \\ https://hal.science/hal-03320517}

Submitted on 16 Aug 2021

HAL is a multi-disciplinary open access archive for the deposit and dissemination of scientific research documents, whether they are published or not. The documents may come from teaching and research institutions in France or abroad, or from public or private research centers.
L'archive ouverte pluridisciplinaire HAL, est destinée au dépôt et à la diffusion de documents scientifiques de niveau recherche, publiés ou non, émanant des établissements d'enseignement et de recherche français ou étrangers, des laboratoires publics ou privés. 


\title{
Linear viscoelasticity of an acrylate IPN, analysis and micromechanics modeling ${ }^{\dagger}$
}

\author{
Julie Diani, $^{a *}$ and Éléonore Strauch-Hausser ${ }^{a}$
}

\begin{abstract}
An amorphous acrylate interpenetrated polymer network (IPN) was made in lab and tested by dynamic mechanical analysis. Using frequency sweep tests, it was shown that the time-temperature superposition principle applies to the double network. Moreover, a generalized Maxwell model with forty Maxwell branches successfully reproduced the material linear viscoelasticity. Using temperature sweep tests, the IPN linear viscoelasticity has been estimated by micromechanics, applying both mean-field homogenization models and fast Fourier transform (FFT)-based homogenization techniques. This modeling effort allowed discussing the simple network mechanical interactions. The microstructure of a second network, defined by a self-avoiding random walk, embedded in a continuous medium, in place of the first network, is shown to provide with satisfactory estimates of the IPN linear viscoelasticity.
\end{abstract}

\section{Introduction}

Interpenetrated polymer networks are intertwined networks built sequentially or with orthogonal chemistry. IPN hydrogels have drawn attention recently [1], especially for their increased toughness using one network as sacrificial bonds [2]. The latter application was also carried on successfully for rubbers [3]. However, IPNs are also interesting for their viscoelastic properties. Actually, an IPN may show a wider glass transition or two glass transitions at tailored temperatures [4], which may benefit applications such as shape memory ones[5].

black The linear viscoelasticity of IPNs has been characterized by dynamic mechanical analysis (DMA) using temperature sweep tests only, and frequency sweep tests have not been reported in the literature. While temperature sweep tests provide information on the phase separation and molecular mobility, frequency sweep tests give access to the mechanical parameters useful to calculate the material linear viscoelastic response for any loading. With frequency sweep tests, the time-temperature superposition principle may be tested. When the latter applies, the shift factors are determined while building the master curves. The relaxation time spectrum is calculated by fitting the master curves. For instance, these mechanical parameters are useful to predict the shape memory of amorphous polymers [6]. When two amorphous networks are considered, time-temperature superposition may be expected for the IPN. However, no experimental proof has been presented so far. Therefore, in the present contribution, the mechanical linear viscoelasticity of the amorphous acrylate IPN is characterized using frequency sweep tests in order to see if the time-temperature superposition principle applies to the double network, and if its viscoelasticity can be reproduced by a standard viscoelastic rheological scheme, such as the generalized Maxwell model.

When submitted to DMA temperature sweeps, IPNs show either a single broad glass transition when both networks are not separated well $[7,8,9]$, or two glass transitions in the case of a clear phase separation[8, 9, 10, 11, 12]. In the latter case, when compared to the single network glass transition temperatures, the double network transition temperatures are usually shifted toward each other reflecting the presence of each network in the other one $[8,9,10,12]$. In the case of strong incompatibility, temperature shifts outside of the temperature range of the single network transitions, have been reported [11] also. The extent of the transition temperature shift depends strongly on the IPN composition: nature and mass ratios of the single networks, presence of additives..., and is difficult to predict. However, temperature sweep tests have been used in [7] for micromechanics calculations in an attempt to reproduce the IPN linear viscoelasticity with the viscoelasticity of each single network as input data. Micromechanics modeling is a relevant tool to better understand the single network mechanical interactions. The work of [7] uses Takayanagi's model [13], based on an inclusion/matrix representation of the heterogeneous material, which does not apply well to the physical representation of all IPNs, especially amorphous acrylate IPNs such as ours. Moreover, Takayanagi's model adds an undesired fitting parameter that varies with the temperature. Therefore, different micromechanics approaches are experimented and confronted to the linear

\footnotetext{
${ }^{0 a}$ Laboratoire de Mécanique des Solides, CNRS UMR 7649, École Polytechnique, Institut Polytechnique de Paris, Route de Saclay, 91128, Palaiseau, France.

0*, Tel: 331693357 90; E-mail: julie.diani@polytechnique.edu.

${ }^{0} \dagger$ Published in Soft Matter (2021) vol. 17, pp7341-7349. https://doi.org/10.1039/d1sm00808k
} 
Table 1: Material compositions

\begin{tabular}{llllll}
\hline Network & $\begin{array}{l}\text { MA } \\
(\text { mass } \%)\end{array}$ & $\begin{array}{l}\text { BA } \\
(\text { mass\%) }\end{array}$ & $\begin{array}{l}\text { MA } \\
(\text { mol \%) }\end{array}$ & $\begin{array}{l}\text { BA } \\
(\text { mol \%) }\end{array}$ & $\begin{array}{l}\text { PEGDMA } \\
(\text { mol \%) }\end{array}$ \\
\hline MA & 100 & - & 97.5 & - & 2.5 \\
BA & - & 100 & - & 95 & 5 \\
IPN1 & 52 & 48 & - & - & - \\
IPN2 & 55 & 45 & - & - & - \\
IPN3 & 30 & 70 & - & - & - \\
\hline
\end{tabular}

viscoelasticity of the acrylate IPN. First, classic mean-field homogenization models based on an inclusion/matrix representation, similar to the representation employed by [7], have been tested. Note that models without fitting parameters are considered. Second, a periodic lattice representation of the IPN has been used. The interest of such a representation stands in the fact that more realistic representations of IPNs may be adopted, such as a classic self avoiding random walk for the design of the second network enclosed in the first one. The homogenization is based on the resolution of the LippmannSchwinger equation by a fast Fourrier transform (FFT)-based method $[14,15]$. The latter method has been gaining interest for two reasons. It provides with a fast substitute to finite element simulations within the linear elasticity/viscoelasticity framework, and it allows to run calculations on complex microstructures characterized by X-ray microtomography for instance, due to the 3D lattice voxel representation of the images. However, to the authors' knowledge it has not been confronted yet to linear viscoelastic experimental data, which is done here.

The paper is organized as follows. Next section presents how the single networks and the IPNs were made and tested. Section 3 shows and discusses the linear viscoelasticity characterization by both frequency sweep and temperature sweep DMA tests. In section 4, micromechanics models are performed in an attempt to reproduce the IPN temperature sweep tests in order to assess the mechanical interactions of the single networks.

\section{Materials and tests}

The amorphous acrylate interpenetrated networks were made in the lab following a procedure found in [16]. The chemical products, monomers butyl acrylate BA (CAS 141-32-2) and Methyl acrylate MA (CAS 96-33-3), crosslinker 550 $\mathrm{g} / \mathrm{mol}$ molar weight poly(ethylene glycol) dimethacrylate (PEGDMA) (CAS 2585-47-5) and photoinitiator 2-dimethoxy-2phenylacetophenone (DMPA) were used as received by Sigma-Aldrich.

For the first network, $97.5 \%$ moles of BA were mixed with 2.5\% moles of PEGDMA and crosslinked for 40 minutes in an ultra violet chamber UVP CL-1000. This simple BA network was crosslinked in between glass plates in order to obtain uniform plates in which rectangular samples were cut. A second mix was prepared with $95 \%$ moles of MA and $5 \%$ mole of PEGDMA. Some of this mix was crosslinked in the UV chamber like the BA network, providing sample of the second simple network denoted MA. The rest of the MA/PEGDMA mix was used to soak rectangular samples cut in the BA network plates. The latter samples were then placed in the UV chamber in between glass plates for 40 minutes in order to crosslink the MA network. black According to the duration of soaking, different mass ratios of BA $v$ s. MA were obtained. Swelling equilibrium were obtained for samples IPN1 and IPN2. Their final dimensions were increased of approximately $25 \%$ in each direction, thickness, width and length, after the second network crosslinking. Along the IPN making process, the solid rectangular samples were weighted a first time before soaking, and a second time after the MA network was crosslinked, in order to estimate the BA $v$ s. MA mass ratios. The compositions of the obtained double networks have been listed in table 1.

Rectangular samples of BA and MA simple networks and the IPN samples were tested in torsion by dynamic mechanical analysis on a MCR 502 rheometer from Anton Paar at a small strain amplitude of $0.1 \%$, giving access to the dynamic shear modulus. Two types of tests were run, frequency sweep tests from 0.01 to $10 \mathrm{~Hz}$ at given temperatures, and temperature sweep tests at constant frequency of $1 \mathrm{~Hz}$ and temperature ramp of $2{ }^{\circ} \mathrm{C} / \mathrm{min}$.

\section{Linear viscoelasticity characterization}

\subsection{Frequency sweep tests}

Frequency sweep tests were run first in order to possibly assess the time-temperature superposition shift factors and the relaxation time spectrum of the double amorphous network. The material IPN1 was submitted to frequency sweeps from 0.01 to $10 \mathrm{~Hz}$ at given temperature ranging from $-55^{\circ} \mathrm{C}$ to $25^{\circ} \mathrm{C}$ with increasing steps of $2.5^{\circ} \mathrm{C}$. Figure 1 shows the storage modulus $\left(G^{\prime}\right)$, loss modulus $\left(G^{\prime \prime}\right)$ and damping factor $(\tan \delta$ ) master curves successfully built by applying time-temperature 

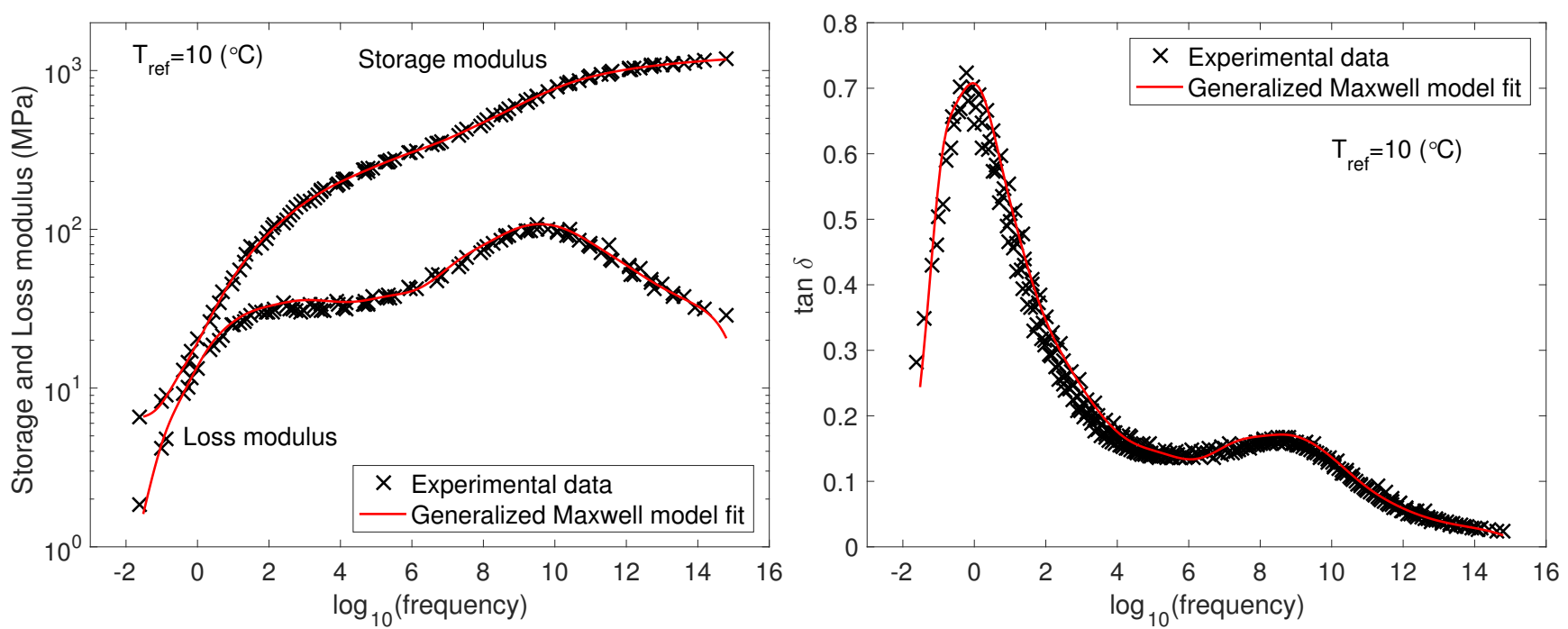

Figure 1: IPN1 shear storage and loss moduli and damping factor $(\tan \delta)$ master curves obtained by time-temperature superposition at reference temperature $10^{\circ} \mathrm{C}$ for torsion DMA frequency sweep tests. The solid lines show the fit of the experimental data by a generalized Maxwell model.

superposition. The damping factor curve exhibits two peaks, indicating a clear phase separation between the two simple networks MA and BA. While clearly visible, the BA peak observed at high frequency, is rather small due to the glassy state of the MA network that prevents the BA network mobility.

The IPN1 shift factors resulting from the master curve building were compared to the shift factors of the single networks. Figure 2 shows the comparison at reference temperatures $10^{\circ} \mathrm{C}$ for the BA network and $-30^{\circ} \mathrm{C}$ for the MA network. The glass transitions of the single networks being significantly spaced out, it was not possible to produce experimental data for both single networks at the same reference temperatures. The values of $\log \left(a_{T}\right)$ with respect to $T$ displayed by single amorphous networks, are usually fitted by the WLF equation[17]:

$$
\log \left(a_{T}\right)=\frac{-C_{1}\left(T-T_{g}\right)}{C_{2}+\left(T-T_{g}\right)}
$$

within the glass transition and by an Arrhenius function (proportional to $1 / T$ ) in the glassy state. The convex part of the shift factors is well recognized on the MA and BA network curves. While still visible, it is less pronounced for IPN1. It is not possible to predict the IPN shift factors, simply knowing the shift factors of both constitutive simple networks since the shift factors are linked to the mobility of each network within its environnement. Note that neither a WLF or an Arrhenius function could fit well the IPN shift factors.

The linear viscoelastic behavior of IPN1 was then successfully fitted by a generalized Maxwell model [18] requiring no less than 40 viscoelastic Maxwell branches (Figure 1). black Note that single acrylate network viscoelasticities are usually very well reproduced by 10 to 20 relaxation times. The Maxwell branch relaxation time spectrum characterizing the material viscoelasticity is displayed in Figure 3. The number of Maxwell branch shows the complexity of the viscoelastic relaxations.

In the end, the frequency sweep tests showed that the IPN verifies the time-temperature superposition principle, and its linear viscoelasticity may be well reproduced by a generalized Maxwell model. black Assessing the linear viscoelastic spectrum and time-temperature superposition parameters, allows simple simulations of the IPN viscoelastic response for any mechanical and thermal loading.

Building master curves at the same reference temperature, for the three networks BA, MA and IPN, is impossible. Actually, the ranges of relevant temperatures for the viscoelasticity characterization of both simple networks do not overlap. Therefore, temperature sweep tests were run in order to provide with input data for micromechanics modeling. These tests are presented in the next section before micromechanics modeling was applied to better understand the single network mechanical interactions within the IPN structure.

\subsection{Temperature sweep tests}

The temperature sweep tests were run at least twice on each samples. Figure 4 presents the storage and loss moduli and the damping factors with respect to the temperature for single networks BA and MA, and for both double networks IPN2 


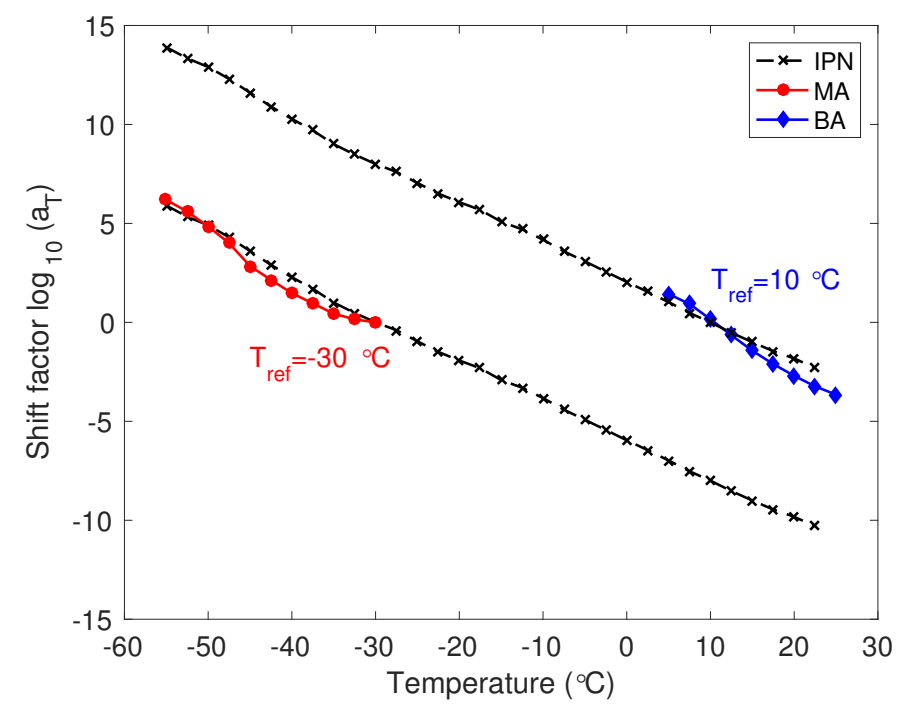

Figure 2: IPN1 shift factors characterizing the double network time-temperature superposition compared to the shift factors of the BA and MA simple networks.

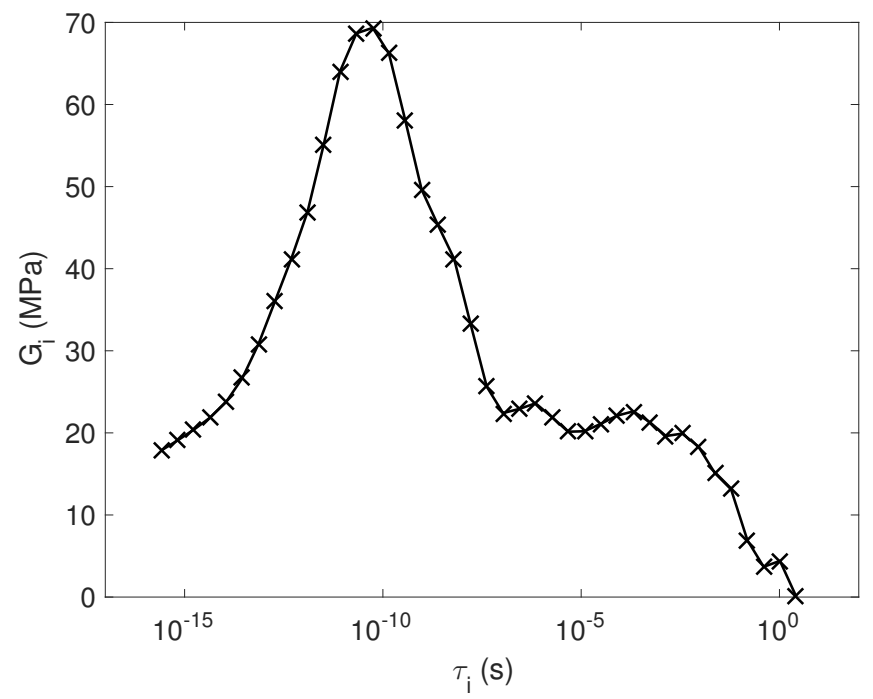

Figure 3: Generalized Maxwell model relaxation time spectrum used for model representation in Figure 1. 

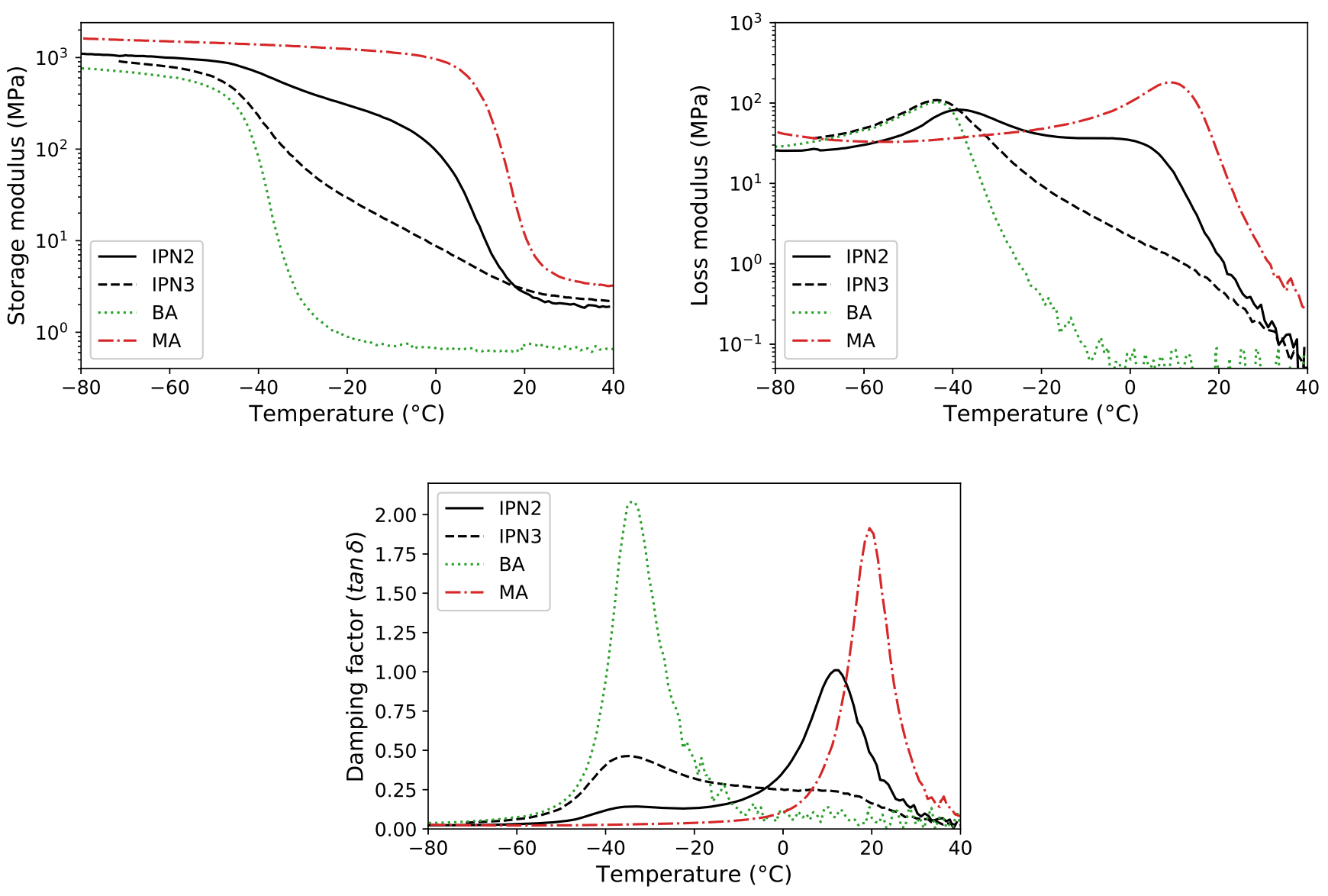

Figure 4: Storage modulus, loss modulus and damping factor of the MA, BA, IPN2 and IPN3 networks measured during torsion DMA temperature sweeps at $2^{\circ} \mathrm{C} / \mathrm{min}$.

and IPN3. Temperature sweep tests are less accurate than frequency sweep tests for the temperature is never perfectly homogeneous in the samples [19]. black In order to limit the discrepancy, sample dimensions were chosen similar and close to $30 \mathrm{~mm}$ length, $7 \mathrm{~mm}$ width and $1 \mathrm{~mm}$ thick for every network.

The MA and BA single networks present a glass transition determined by their loss modulus peaks at -43.6 and $9.1^{\circ} \mathrm{C}$ respectively. The IPNs show two phase transitions, the first one being due to the increased mobility of the BA network, while the second transition occurs when the MA network state changes from glassy to rubbery. The temperatures at which each transition occurs are not necessarily the same as the single network glass transition temperatures. For instance, samples of IPN2 display a shift of approximately $6^{\circ} \mathrm{C}$ toward the higher temperatures for the BA network and of $7^{\circ} \mathrm{C}$ toward the lower temperatures for the MA network compared to the single network glass transition temperatures. Interpenetrated polymer network IPN3 shows no shift of the BA network glass transition while the MA network transition seems shifted like in IPN2. Such shifts of the single network transition temperatures have been observed commonly in INPs, see for instance $[8,9,11]$. However, the extent of the transition temperature shifts depends on various parameters such as the nature of the simple networks, their mass ratios, their miscibility, the presence of additives, whether IPNs or semi-IPNs are considered. As an example, the impact of the single network mass ratios is illustrated by the different shifts observed in IPN2 and IPN3.

In terms of viscoelastic characterization, the interest of the experimental data shown in Figure 4, stands in the fact that for a temperature range covering both transitions of the amorphous IPN network, experimental data of the storage and loss moduli of both BA and MA networks are known. They may be used as input in micromechanics models to compute the theoretical IPN network storage and loss moduli with respect to temperature. Comparisons between the theoretical estimates and the experiments will help to decide on the appropriate physical representation of the IPN that correctly capture the mechanical interactions of both simple networks within the IPN. 


\section{Micromechanics analysis}

\subsection{Models}

The IPNs may be seen as heterogeneous materials, the BA and MA networks being the constitutive phases. The IPN linear viscoelasticity results from the linear viscoelasticity of both constitutive phases, the volume ratios of BA $v$ s. MA and how both single networks interact. We aim at finding out the latter using a multi-scale approach.

black Micromechanics modeling is used to estimate the material properties of the equivalent homogeneous material to a heterogeneous material. Considering the linear elastic response of the heterogeneous IPN, the constitutive equations of its equivalent homogeneous material may write as:

$$
\Sigma=\mathbf{C}_{h}: \mathbf{E}
$$

with $\mathbf{C}_{h}$ the stiffness tensor and $\Sigma$ and $\mathbf{E}$ the average stress and strain tensors defined as,

$$
\Sigma=\int_{V} \sigma(x) d V, \quad \mathbf{E}=\int_{V} \varepsilon(x) d V
$$

$\sigma$ and $\varepsilon$ are the local stress and strain tensors defined at any material point $x$. Two general approaches of micromechanics modeling will be applied allowing various representations of the heterogeneous material.

First, the two-phase material is represented as spherical inclusions for one phase, embedded in a matrix for the other phase. With this representation, mean-field homogenization based on the Eshelby's solution of the single inclusion problem [20] may be used, and constitutive equations write as,

$$
\mathbf{C}_{h}=\mathbf{C}^{m}+f\left(\mathbf{C}^{i}-\mathbf{C}^{m}\right): \mathbf{A}
$$

with $\mathbf{C}^{m}$ and $\mathbf{C}^{i}$ the stiffness tensors of the phases acting as matrix and inclusion, $f$ the volume ratio of inclusions, and $\mathbf{A}$ the concentration-strain tensor depending on the chosen micromechanical model. Note that for an isotropic material, the tensor $\mathbf{C}$ components write simply in terms of the material bulk modulus $K$ and the shear modulus $G$ as,

$$
C_{i j k l}=K \delta_{i j} \delta_{k l}+G\left(\delta_{i k} \delta_{j l}+\delta_{i l} \delta_{j k}-\frac{2}{3} \delta_{i j} \delta_{k l}\right)
$$

The Mori-Tanaka [21] and generalized self-consistent (GSC) [22] models are considered, as well as the Voigt and Reuss bounds. These bounds assume homogeneous strain or stress in both phases and simply write as,

$$
\mathbf{C}_{h}=\mathbf{C}^{m}+f\left(\mathbf{C}^{i}-\mathbf{C}^{m}\right),
$$

for the Voigt bound and,

$$
\mathbf{S}_{h}=\mathbf{S}^{m}+f\left(\mathbf{S}^{i}-\mathbf{S}^{m}\right)
$$

for the Reuss bound with $\mathbf{S}=\mathbf{C}^{-1}$. On one hand, the Mori-Tanaka model was chosen for its simple explicit expression. It has been designed for fillers surrounded by a continuous matrix and applies better for inclusion volume ratios below $30 \%$. On the other hand, the implicit generalized self-consistent model takes better into account the interactions between particles. It has been shown to reproduce well the mechanical behaviors of matrices with randomly dispersed spherical inclusions $[23,24]$. Models and bounds of interest present analytical solutions in linear elasticity, and therefore extend to the linear viscoelasticity, by merely replacing the elastic constant (for instance $G$ ) by their complex expression $\left(G^{*}=G^{\prime}+j G^{\prime \prime}\right.$ ), thanks to the elastic-viscoelastic correspondence principle from Hashin[25].

Second, two periodic cubic lattice representations of the double network are adopted. First, voxels are set to behave as one or the other phase randomly, within the only constraint of reaching the target volume ratio of each phase (Figure 5a). Second, starting from one seed voxel, one phase is built continuously by a self-avoiding random walk, creating a worm-like phase within a continuous phase (Figure 5b). Assuming periodic boundary conditions, the mechanical problem defining the equivalent homogeneous behavior (Eq. 2), and which writes as:

$$
\left\{\begin{array}{l}
\sigma(x)=\mathbf{C}(x): \varepsilon(x) \\
\operatorname{div} \sigma(x)=0 \\
\varepsilon(x)=\frac{1}{2}\left(\nabla u(x)+\nabla^{T} u(x)\right) \\
\mathbf{E}=\int_{V} \varepsilon(x) d V
\end{array}\right.
$$

is equivalent to the Lippmann-Schwinger equation,

$$
\left(\mathbf{I}+\Gamma^{0} * \delta \mathbf{C}\right): \varepsilon=\mathbf{E}, \quad \delta \mathbf{C}(x)=\mathbf{C}(x)-\mathbf{C}^{0}
$$




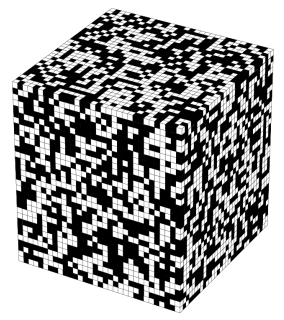

(a)

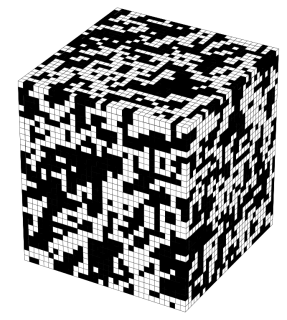

(b)

Figure 5: Material representation by periodic cubic lattice microstructures for the FFT-based homogenization (a) Random distribution of both phases. (b) Use of a random walk to build a worm-like phase enclosed in a continuous phase.

with $\Gamma^{0}$ the Green operator associated to the constant stiffness $\mathbf{C}^{0}$ of an homogeneous reference material, which is conveniently chosen isotropic. Such an equation can be advantageously solved in the Fourier space giving rise to the FFT-based homogenization scheme $[14,15]$, which increasing popularity stands in the fast resolution provided by the FFT method and the grid representation of the heterogeneous material, well fitted for microstructure characterization by computerized tomography scans. The operator $\Gamma^{0}$ writes in the Fourier space as:

$$
\begin{aligned}
\Gamma_{i j k l}^{0}(\xi) & =\frac{1}{4 G_{0}|\xi|^{2}}\left(\delta_{k i} \xi_{l} \xi_{j}+\delta_{k j} \xi_{l} \xi_{i}+\delta_{l i} \xi_{k} \xi_{j}+\delta_{l j} \xi_{k} \xi_{i}\right) \\
& -\frac{\lambda_{0}+G_{0}}{G_{0}\left(\lambda_{0}+2 G_{0}\right)} \frac{\xi_{i} \xi_{j} \xi_{k} \xi_{l}}{|\xi|^{4}}
\end{aligned}
$$

with $\lambda_{0}=K_{0}-\frac{2 G_{0}}{3}, G_{0}$ and $K_{0}$ being the shear and bulk moduli characterizing the reference material. The behaviors of the equivalent homogeneous IPNs described by microstructures (Figure 5) are then obtained with an accelerated resolution [26] of algorithm [14, 15] written in the context of linear viscoelasticity (Algorithm 1). The linear viscoelasticities of the single networks are taken into account by replacing the elastic stiffness tensor $\mathbf{C}$ (Eq. 5) by the complex stiffness tensor $\mathbf{C}^{*}$ calculated similarly with the complex moduli $K^{*}$ and $G^{*}$. Note that the complex problem (Algorithm 1 ) is solved simply by choosing an elastic reference material $\mathbf{C}^{0}$ with Eq. (10) remaining.

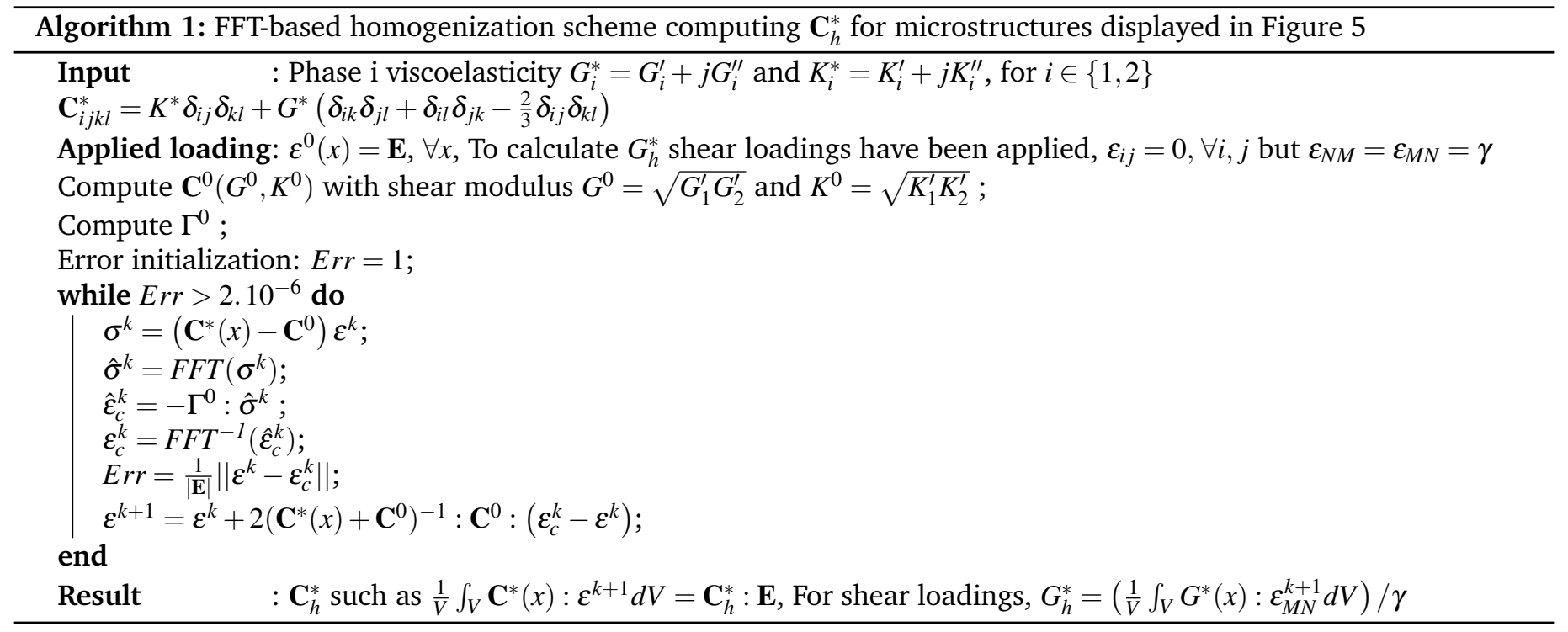

Cubic grids from $32^{3}$ to $192^{3}$ voxels were tested. Obviously, as the cube size increases, the microstructure isotropy improves and the difference of behaviors between two similar (random or worm-like) microstructures reduces. However, the difference of shear modulus between microstructures of $192^{3}$ voxels and microstructures of $128^{3}$ voxels was less than $0.005 \%$. Moreover, due to the randomness of the microstructures considered here, even small grids as $32^{3}$ could give access to the shear modulus with a reasonable $2 \%$ underestimate, when its value was estimated by averaging the results on five microstructures. In the end, after validating the excellent isotropy of the $128^{3}$ voxels microstructures, results presented here were obtained on one microstructure of $128^{3}$ voxels microstructure submitted to one shear loading. 

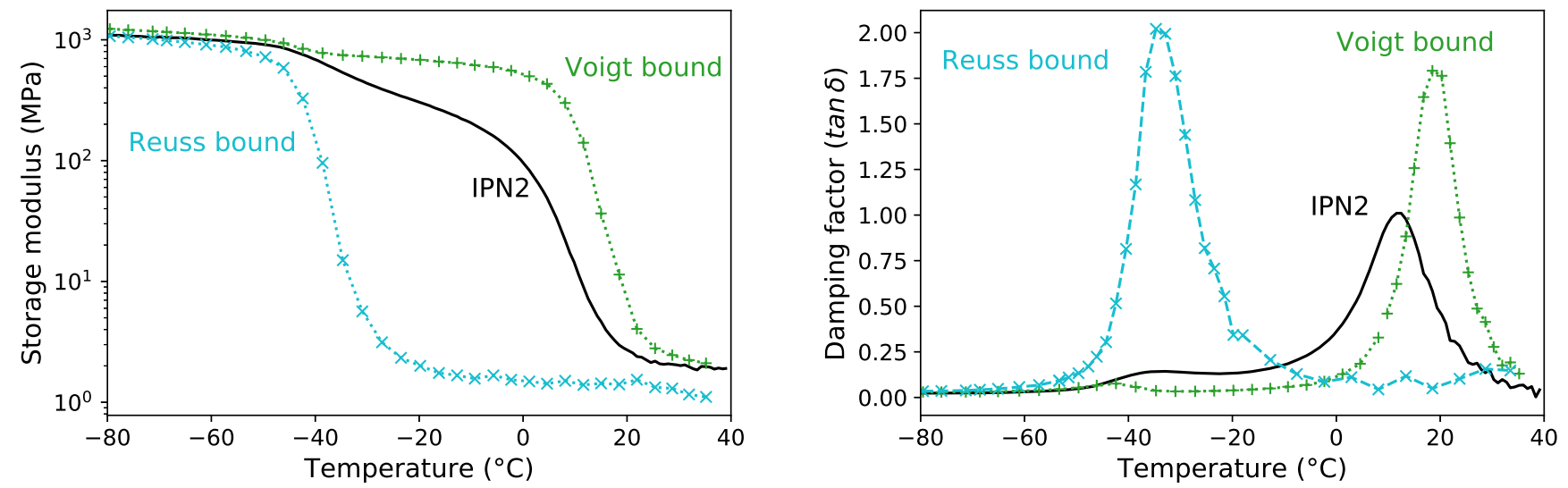

Figure 6: Voigt and Reuss bounds for IPN2 containing 45\% of BA and 55\% of MA.

\subsection{Results and discussion}

The storage and loss shear moduli defining the complex shear moduli of networks BA and MA have been characterized experimentally. In order to run three dimensional computations as required by the chosen homogenization models, the bulk modulus $K$ has been assumed elastic constant and equal to a reasonable value of $3000 \mathrm{MPa}$ for both MA and BA networks. Actually, accounting for a more realistic viscoelastic bulk modulus adds complexity, since the experimental characterization is very complex, without changing noticeably the shear modulus of the equivalent heterogeneous material since the drop of the bulk modulus upon the glass transition is of a factor two or three only.

black First, the simple Voigt and Reuss bounds, which do not require any microstructure representation, are calculated for $45 \%$ of BA corresponding to the IPN2 composition. Figure 6 shows the comparison between the theoretical estimates and the IPN2 behavior when the MA and BA network behaviors are considered without any glass transition shift as witnessed in Figure 4. While not ideal, the Voigt bound seems closer to a realistic representation of the IPN. These unsatisfactory results show that in the IPN, stresses and strains supported by both networks are different. Moreover, the theoretical IPN transition temperatures are misplaced compared to the actual IPN, which was to be expected upon remarks made in section 3.2. In order to better match the temperatures of transitions observed for IPN2 network, the Voigt bound has been recalculated considering the glass transition shifts of +6 and $-7^{\circ} \mathrm{C}$ noticed for the BA and MA in Figure 4. Figure 7 shows how the model representation improves when accounting for the temperature shifts. Without accounting for the latter, no model can provide satisfactory comparisons with the actual materials. Therefore, in the following, the input data are shifted of $+6^{\circ} \mathrm{C}$ for the BA and $-7^{\circ} \mathrm{C}$ for the MA when IPN2 behavior is calculated and of $-7^{\circ} \mathrm{C}$ for the MA only when IPN3 is considered. Last with the bounds, the Voigt and Reuss bounds have been tested for IPN3 in Figure 8. The result is unsatisfactory and strengthens the idea that interactions between both networks are actually more complex than a mere homogeneous stress or strain assumption.

Figure 9 shows the storage modulus and damping factor calculated with the Mori-Tanaka and generalized self-consistent models for IPN2. The results for the mean-field homogenization schemes were calculated considering BA inclusions in an MA surrounded matrix. It is worth noting that when the MA network was considered as the inclusion phase and the BA as the matrix one, model viscoelastic responses were closer to the Reuss bound. The inclusion/matrix representation appears as unsatisfactory for IPNs. While, this could be easily expected, this representation was the only one adopted in the previous attempt of IPN viscoelasticity modeling [7]. The Takayanagi's model [13], initially designed for semi-crystalline materials, was applied and showed rather encouraging results. However, this was due to an unphysical fitting parameter that artificially changed the amount of each phase contributing to the heterogeneous material viscoelasticity with respect to temperature.

Last, FFT-based homogenization was run for both random and worm-like microstructures. Figure 10 shows the comparison between the IPN2 and the theoretical estimates. Both microstructures provide with similar rather remarkable results without adding any fitting parameters. Note that the BA network was chosen as the continuum medium in the worm-like representation. However, since the volume ratio of BA vs. MA is close to 0.5, considering either the MA or the BA network as the continuum medium drives to similar results. Therefore, in order to further test both random and worm-like representations, a comparison with IPN3 viscoelasticity is shown in Figure 11. One notices that the worm-like representation produces much better results. The latter were obtained for the BA network acting as the continuum medium in the worm-like representation, when considering otherwise, results were much less satisfactory, the highest peak of $\tan \delta$ 

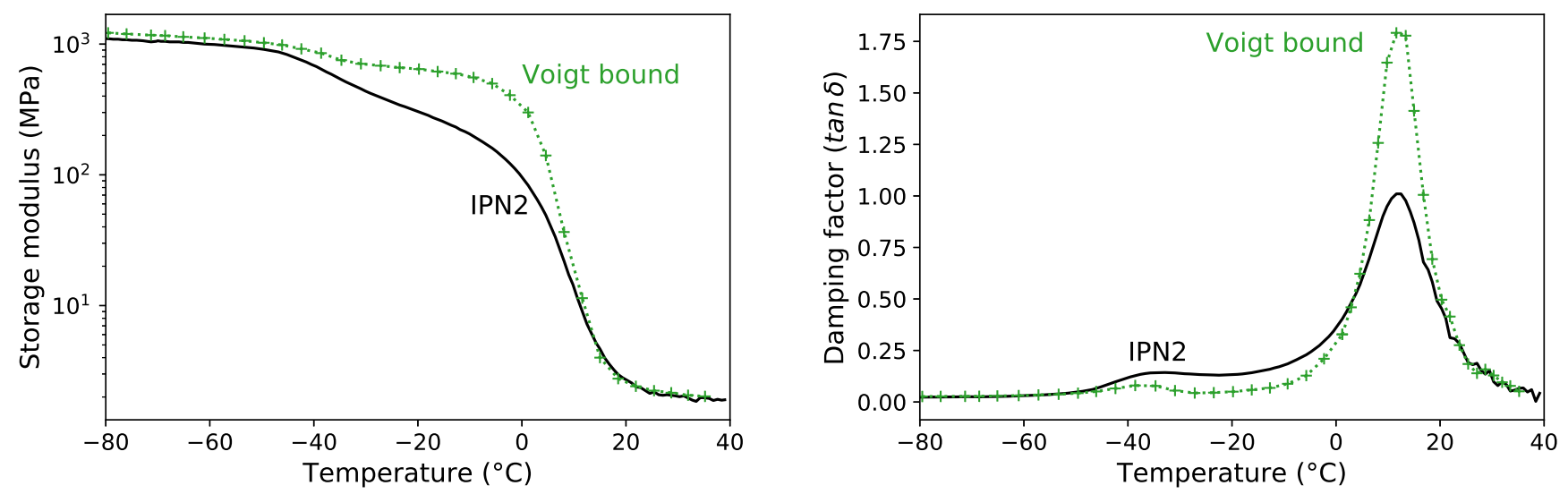

Figure 7: Voigt bound estimated for IPN2 when the BA and MA input viscoelasticity have been shifted of +6 and $-7^{\circ} \mathrm{C}$ respectively.
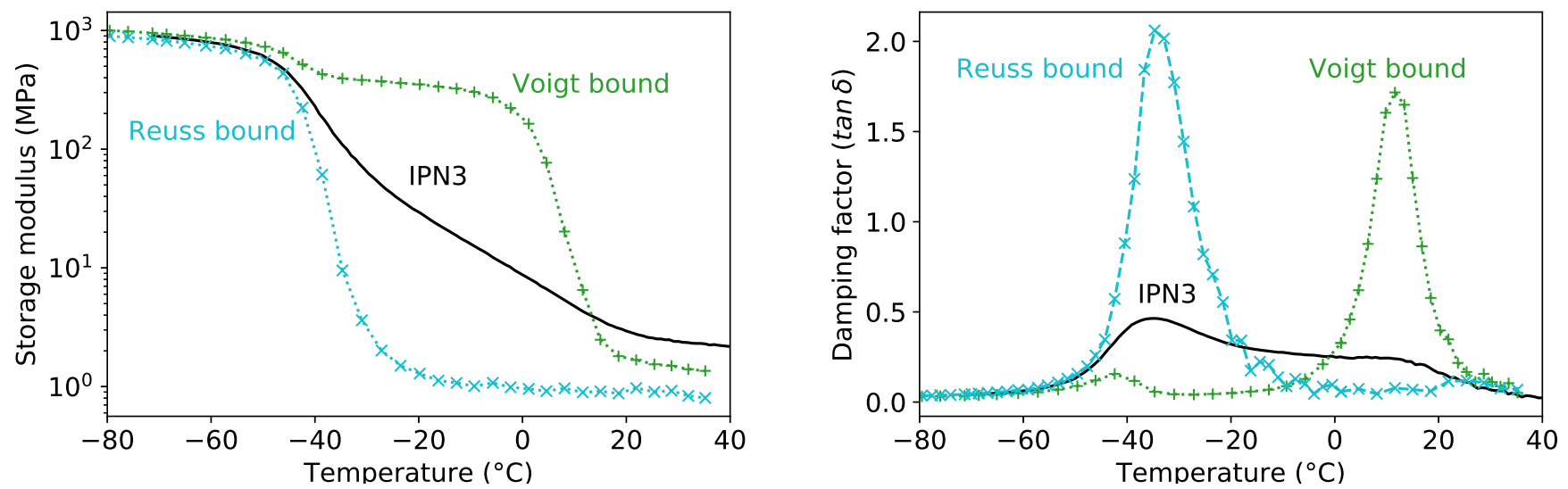

Figure 8: Voigt and Reuss bounds for IPN3 containing 70\% of BA and 30\% of MA when the MA input viscoelasticity have been shifted of $-7^{\circ} \mathrm{C}$. 

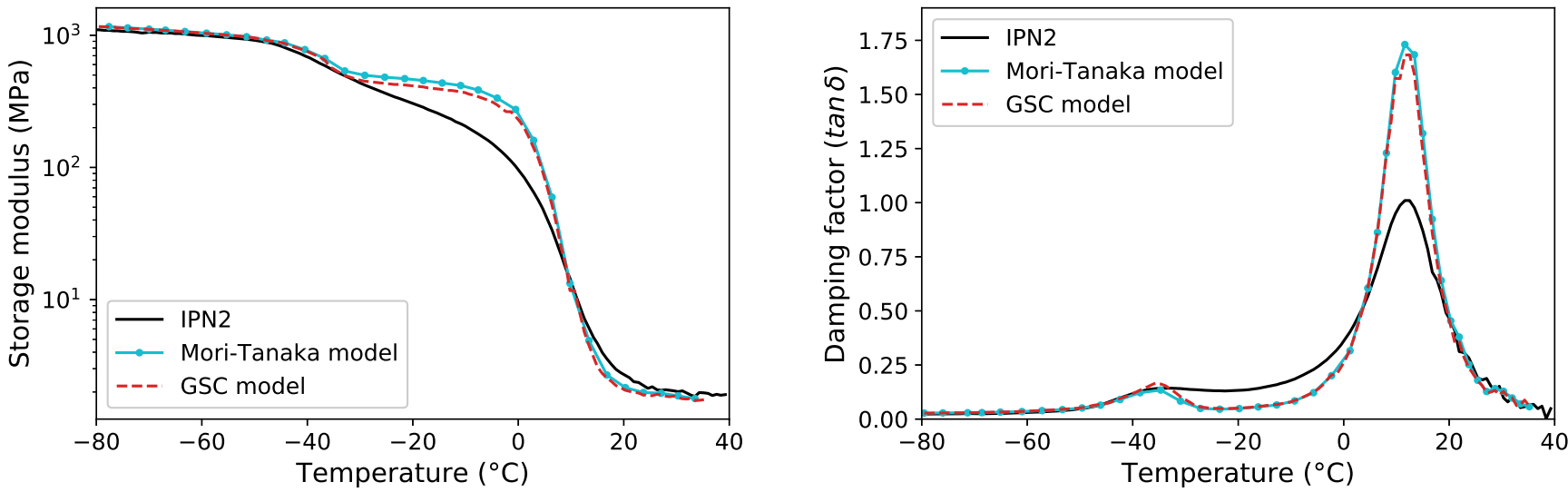

Figure 9: Comparison between IPN2 linear viscoelasticity and the Mori-Tanaka and generalized self-consistent estimates when the BA and MA input viscoelasticities have been shifted of +6 and $-7^{\circ} \mathrm{C}$ respectively.
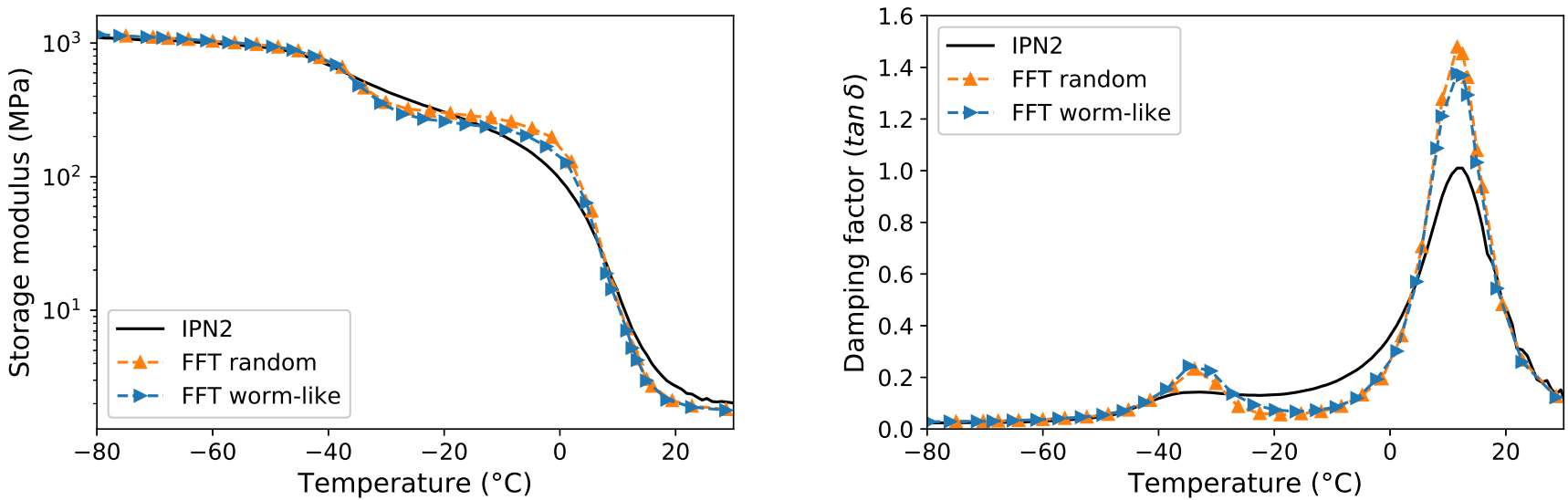

Figure 10: Comparison between the linear viscoelasticity of IPN2 and its representation by a worm-like and a random microstructure calculated by FFT-homogenization scheme when the BA and MA input viscoelasticities have been shifted of +6 and $-7^{\circ} \mathrm{C}$ respectively.

being obtained at the higher temperatures like for IPN2. These results show that the physically sounded worm-like representation, defining the second single network as a self-avoiding random walk built in a continuum medium representing the first single network, is the best representation to reproduce the mechanical interactions of the single networks when intertwined in the IPN.

\section{Conlusion}

Acrylate amorphous interpenetrated networks have been prepared in the lab for in depth linear viscoelastic characterization. The dynamic mechanical analysis run with frequency sweep tests, provided with several original results. First, the amorphous IPN was shown to satisfy to the time-temperature superposition principle. Second, the material linear viscoelasticity could be well reproduced by a generalized Maxwell model as long as enough viscous Maxwell branches were taken into account.

Temperature sweep tests have been useful to apply homogenization modeling in order to reproduce the double network linear viscoelasticity. As for other IPNs characterized in the literature, it was noticed a shift of the glass transition temperature of each simple network when interlocked in the IPN. Unfortunately, these shifts are difficult to predict for they depend on various physical and chemical parameters, black as it was shown for the volume ratios of both single networks. The glass transition temperature shifts were experimentally estimated and taken into account to compute the IPN linear viscoelasticity using two micromechanics approaches black in an attempt to better understand the single net- 

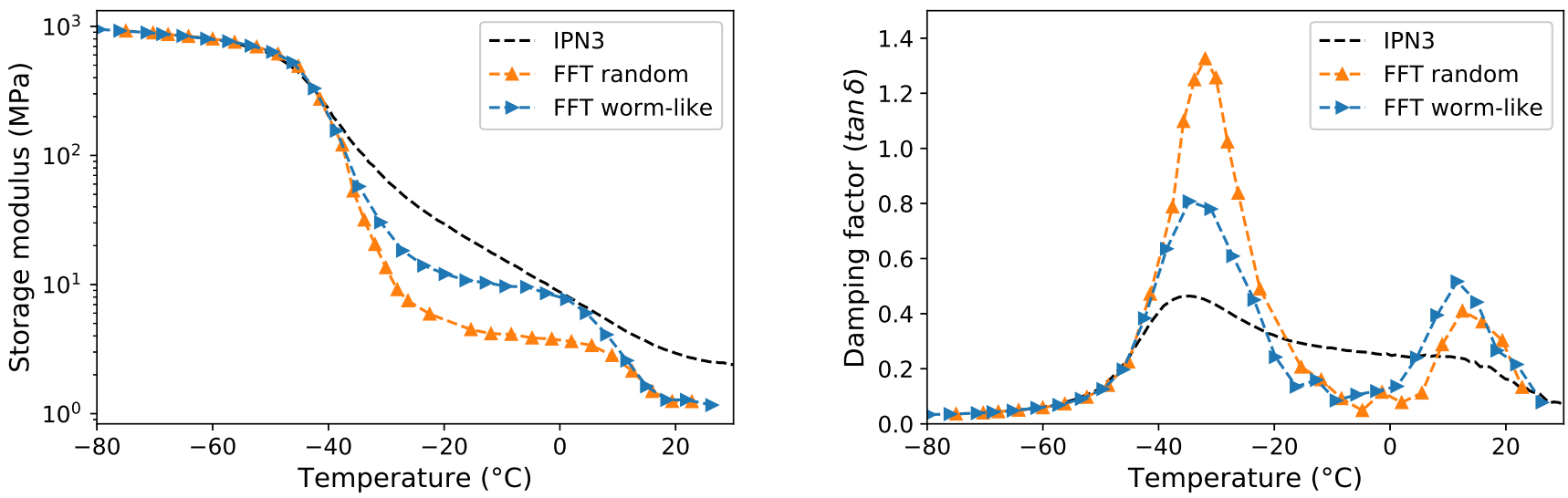

Figure 11: Comparison between the linear viscoelasticity of IPN3 and its representation by a worm-like and a random microstructure calculated by FFT-homogenization scheme when the MA viscoelasticity has been shifted of $-7^{\circ} \mathrm{C}$.

work mechanical interactions. The actual material viscoelasticity was shown to be well reproduced when representing the double network by a cubic grid where the first crosslinked network is a continuous medium and the second crosslinked network is a worm-like network built in the continuum medium. The relevance of such a representation may now be extended past linear viscoelasticity to predict the mechanical response of such materials. The relevance of the modeling approach may be extended to more complex network microstructures.

\section{Acknowledgements}

Dr. Diani is thankful to Dr. Renald Brenner for helpful discussions and validation tests for the FFT-based homogenization.

\section{Author Contributions}

Lead researcher: Dr. Diani ran some of the temperature sweep tests, computed the generalized Maxwell model parameters and the micromechanics models, and wrote the paper.

Supporting: Bachelor student E. Strauch-Hausser successfully made IPNs in lab (which was never done in our lab), ran DMA frequency sweeps tests and built master curves, ran some of the temperature sweep tests.

\section{References}

[1] E.S. Dragan. Design and application of interpenetrating polymer network hydrogels. a review. Chem. Eng. J., 243:572-590, 2014.

[2] J.P. Gong, Y. Katsuyama, T. Kurokawa, and Y. Osada. Double-network hydrogels with extremely high mechanical strength. Adv. Mater., 15:1155-1158, 2003.

[3] E. Ducrot, Y Chen, M. Butlers, R.P. Sijbesma, and C. Creton. Toughening elastomers with sacrificial bonds and watching them break. Science, 344:186-189, 2014.

[4] L.H. Sperling. Interpenetrating polymer networks, an overview. Pergamon, 1996.

[5] Y. Wu, J. Hu, J. Han, Y. Zhu, H. Huang, J. Li, and B. Tang. Two-way shape memory polymer with "switching-spring" composition by interpenetrating polymer network. J. Mater. Chem. A, 31:18816-18822, 2014.

[6] J. Diani, P. Gilormini, C. Frédy, and I. Rousseau. Predicting thermal shape memory of crosslinked polymer networks from linear viscoelasticity. Int. J. Solids and Struct., 49:793-799, 2012.

[7] V.F. Rosovizky, M. Ilavský, J. Hrouz, K. Dušek, and S. Yu. Viscoelastic behavior of interpenetrating networks of polyurethane and polyurethane acrylate. J. Appl. Polym. Sci., 24:1007-1015, 1979. 
[8] J. M. Meseguer Dueñas, D. Torres Escuriola, G. Gallego Ferrer, M. Monleón Pradas, J.L. Gómez Ribelles, P. Pissis, and A. Kyritsis. Miscibility of poly(butyl acrylate)-poly(butyl methacrylate) sequential interpenetrating polymer networks. Macromolecules, 34:5525-5534, 2001.

[9] N.R. Manoj, D. Ratna, V. Dalvi, L. Chandrasekhar, M. Patri, B.C. Chakraborty, and P.C. Deb. Interpenetrating polymer networks based on carboxylated nitrile rubber and poly(alky methacrylate)s. Polym. Eng. Sci., 42:1748-1755, 2002.

[10] A.P. Mathew, G. Groeninckx, G.H. Michler, H.J. Radushch, and S. Thomas. Viscoelastic properties of nanostructured natural rubber/polystyrene interpenetrating polymer networks. J. Polym. Sci. Part B, 41:1680-1696, 2003.

[11] N.V. Babkina, Y.S. Lipatov, and T.T. Alekseeva. Damping properties of composites based on interpenetrating polymer networks formed in the presence of compatibilizing additives. Mech. Comp. Mater., 42:385-392, 2006.

[12] K.M. Dean, W.D. Cook, and M.Y. Lin. Small angle neutron scattering and dynamic mechanical thermal analysis of dimethacrylate/epoxy ipns. Eur. Polym. J., 42:2872-2887, 2006.

[13] M. Takayanagi, S. Uemura, and S. Minami. Application of equivalent model method to dynamic rheo-optical properties of crystalline polymer. J. Polym. Sci. C., 5:113-122, 1964.

[14] H. Moulinec and P. Suquet. Fa fast numerical method for computing the linear and nonlinear properties of composites. C. R. Acad. Sci. II, 318:1417-1423, 1994.

[15] Moulinec H. and P. Suquet. A numerical method for computing the overall response of nonlinear composites with complex microstructure. Comp. Meth. Appl. Mech. Eng., 157:69-94, 1998.

[16] E. Ducrot and C. Creton. Characterizing large strain elasticity of brittle elastomeric networks by embedding them in a soft extensible matrix. Adv. funct. Mater, 26:2482-2492, 2016.

[17] M.L. Willimas, R.F. Lande, and J.D. Ferry. The temperature dependence of relaxation mechanisms in amorphous polymers and other glass-forming liquids. J. Amer. Chem. Soc.., 77:3701-3707, 1955.

[18] I.M. Ward and J. Sweeney. An introduction to the mechanical properties of solid polymers. John Wiley and Sons, 2nd edition, 2004.

[19] J. Diani and P. Gilormini. On necessary precautions when measuring solid polymer linear viscoelasticity with dynamic analysis in torsion. Polym. Test., 63:275-280, 2017.

[20] J.D. Eshelby. The determination of the elastic field of an ellipsoidal inclusion and related problems. Proc. Roy. Soc. London, 241:376?396, 1957.

[21] T. Mori and K. Tanaka. Average stress in matrix and average elastic energy of materials with misfitting inclusions. Acta Metal., 21:571-574, 1973.

[22] R.M. Christensen and K.H.. Lo. Solution for effective shear properties in three phase sphere and cylinder models. $J$. Mech. Phys. Solids, 27:315-330, 1979.

[23] A.A. Gusev. Controlled accuracy finite element estimates for the effective stiffness of composites with spherical inclusions. Int. J. Solids Struct., 80:227-236, 2016.

[24] F. de Francqueville, P. Gilormini, and J. Diani. Representative volume elements for the simulation of isotropic composites highly filled with monosized spheres. Int. J. Solids Struct., 158:277-286, 2019.

[25] Z. Hashin. Complex moduli of viscoelastic composites. i. general theory and application to particulate composites. Int. J. Solids Struct, 6:5539-552, 1970.

[26] D. Eyre and G. Milton. A fast numerical scheme for computing the response of composites using grid refinement. Eur. Phys. J., 6:41-47, 1999. 